\title{
Optically thin palladium films on silicon-based substrates and nanostructure formation: effects of hydrogen
}

\author{
Andreas Othonos ${ }^{\mathrm{a}, *}$, Kyriacos Kalli ${ }^{\mathrm{a}}$, Din Ping Tsai ${ }^{\mathrm{b}}$ \\ a Department of Physics, Faculty of Pure and Applied Sciences, University of Cyprus P.O. Box 20537, 1678, Nicosia, Cyprus \\ ${ }^{\mathrm{b}}$ Department of Physics, National Taiwan University, Taipei 10617, Taiwan
}

Received 12 November 1999; accepted 25 January 2000

\begin{abstract}
Optically thin palladium films evaporated on different silicon-based substrates are investigated following exposure to different concentrations of hydrogen gas in air. Laser modulated reflectance off the palladium surface of silicon oxide, silicon nitrite and polycrystalline silicon substrates is used to recover information regarding changes in optical properties of the samples due to the absorption of hydrogen. Simple index of refraction arguments are sufficient to explain the results. Structural changes of the palladium films have been investigated using atomic force microscopy before and after hydrogen exposure. An interesting nanostructure formation is evident in some of the samples, leading to a possible means of fabricating nanodevices. (C) 2000 Elsevier Science B.V. All rights reserved.
\end{abstract}

PACS: $61.16 \mathrm{Ch} ; 68.35 . \mathrm{B} ; 66.55 . \mathrm{Jk} ; 78.66 ; 82.65 . \mathrm{P}$

Keywords: Nanostructures; Optically thin films; Palladium; Silicon-based substrates; Atomic force microscopy; Hydrogen

\section{Introduction}

Over the past few decades, there has been a great deal of interest in investigating the hydrogen-palladium system. This interest has risen from the use of palladium as a catalyst, which results from its property of being able to absorb up to 900 times its own volume in hydrogen. Hydrogen absorption results in structural changes in the metal through the formation of palladium hydride. This process is driven by surface adsorption, through chemisorption, followed by absorption through the palladium volume. One of the most interesting characteristics in a

\footnotetext{
* Corresponding author.
}

hydrogen-palladium system is the transition from the $\alpha$ to $\beta$ phase. Initially, with no hydrogen present, palladium is considered to be in the $\alpha$ phase, however as hydrogen is adsorbed/absorbed, the system passes through a mixed phase region and finally to the $\beta$ phase [1]. The lattice constant changes as hydrogen enters the metal with a maximum change reaching $3 \%$ in the $\beta$ phase. This process is considered reversible, thus as the hydrogen is allowed to escape, the metal system returns to its original state.

The primary interest in most of the work in the past for the hydrogen-palladium system has been for thick palladium films (in excess of $100 \mathrm{~nm}$ ). Optically thick films experience a modification of their surface properties resulting from chemisorption and hence a change in the Fresnel coefficients at the 
surface, this in turn leads to changes in reflectivity. Jansson et al. [2] have shown using ellipsometry that the optical properties of the surface of thick $\sim 180$ $\mathrm{nm}$ Pd films change upon exposure to hydrogen and oxygen. They detected both reversible changes due to hydrogen exposure and irreversible changes related to the poisoning of the metal surface.

Although the bulk behaviour of the palladiumhydrogen system is well characterized, very little work has been done for thin metal films of thickness less than a few tens of nanometers. Abnormal and interesting properties are expected at this film thickness since outer surface effects and changes in optical thickness are complicated by the influence of the second boundary layer at the insulator-metal interface and by the morphological changes of the optically thin metal. Any change that may occur, on exposure of the palladium system to hydrogen gas, is a combination of the surface effects plus index of refraction changes of the bulk.

With the revolution of semiconductor technology and silicon-based integration of most devices, its importance from both an application and fundamental point of view to investigate palladium metal films evaporated on silicon substrates. Even more important is to study optically thin metal films, which offer additional properties arising from structural changes on nanometer scales. Recently, we have used optical techniques to investigate the behavior of several optically thin palladium metal films evaporated on silicon oxide substrate [3].

Here, we will further investigate the exposure to hydrogen of palladium metal thin films evaporated on various silicon-based substrates. Specifically, various optically thin films of palladium evaporated on silicon dioxide, silicon-nitride and polycrystalline silicon are studied following exposure to hydrogen. Of particular interest are the surface structural changes observed in palladium, formed following hydrogen exposure. These changes constitute palladium clusters of nanometer scale, which are examined using an atomic force microscope (AFM).

\section{Experimental details}

The optically ultra-thin samples consist of palladium evaporated onto different silicon substrates (wafers p-type $5 \Omega \mathrm{cm}$ ). The silicon wafers were processed according to standard methods used to obtain gas sensitive catalytic metal-oxide-silicon devices [4]. The silicon dioxide wafers were oxidized in dry oxygen at $1100^{\circ} \mathrm{C}$ to a thickness of $150 \mathrm{~nm}$, the process was followed by a 15-min anneal in argon at the same temperature. The palladium films were deposited by thermal evaporation at a background pressure of about $10^{-7}$ Torr, for a deposition rate of $2-3 \AA / s$ for all film thicknesses. The samples were kept at room temperature during the evaporation and no annealing of the film was performed before the measurements were made. The silicon nitride $\left(\mathrm{Si}_{3} \mathrm{~N}_{4}\right)$ films were grown to a thickness of $50 \mathrm{~nm}$ on a thermally grown oxide in dry oxygen also of $50 \mathrm{~nm}$ thickness, by low pressure CVD. Polycrystalline silicon was also deposited by low pressure CVD to a thickness of $2.3 \mu \mathrm{m}$.

Initially, the reflectivity at $632 \mathrm{~nm}$ of all samples was monitored at room temperatures in a dust-free air environment. These measurements were carried out over a period of several weeks with no evidence of changes in reflectivity. This is to be expected since palladium is stable and forms an oxidization layer only at elevated temperatures (above $80^{\circ} \mathrm{C}$ ). In view of this fact, it was possible to carry out experiments at normal air environment conditions without the unwonted oxidization effects.

Changes in the optical properties of the palladium films following the introduction of hydrogen were monitored using a modulated laser beam reflected off the material surface. This approach has the advantage of being completely passive and it is non-destructive in nature given that the optical power of the probe beam was less than $1 \mathrm{~mW}$. The experimental arrangement is shown in Fig. 1. The thin film palladium samples were placed in a closed cell system with suitable input and output gas ports. These ports were connected to a sensitive gas flow meter system, which provided a control exposure of hydrogen gas to the ultra-thin palladium samples. The incoming $\mathrm{He}-\mathrm{Ne}$ laser beam was modulated with an optical chopper and directed into the closed cell through a window at near normal incidence. The incident beam was approximately $3 \mathrm{~mm}$ in diameter allowing us to sample over a relatively large area of the palladium films. The reflection from the surface was monitored with a silicon detector, at the modulated frequency 


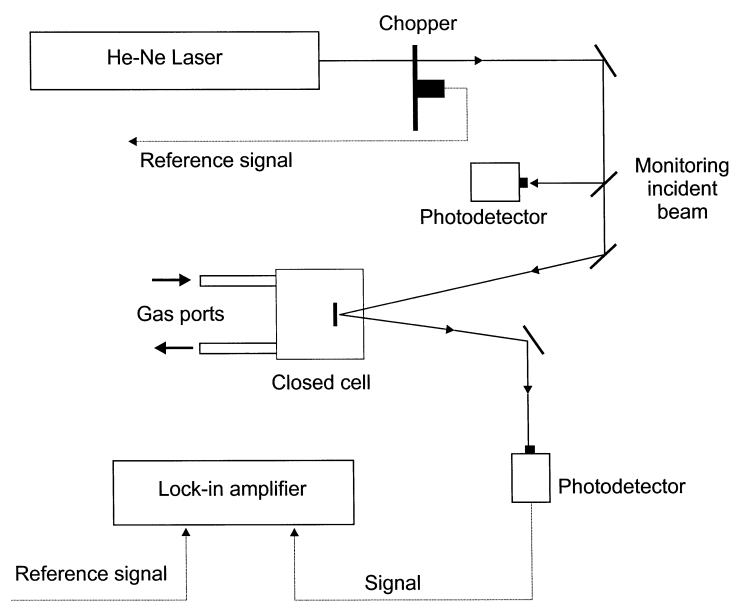

Fig. 1. Experimental setup for measurements of the hydrogen response from palladium optically thin samples.

with a lock-in amplifier. Using the gas system, a precise flow of hydrogen was allowed to enter the cell and expose the sample under investigation. The reflectivity was continuously monitored at room temperature $\left(22^{\circ} \mathrm{C}\right)$ and once saturation was reached, air was introduced into the chamber. This allowed the recovery of the reflectivity signal to its original value.

\section{Results and discussion}

Initially, the hydrogen/air cycling was investigated at the highest concentration (4\% hydrogen) with repeated cycling. The reflectivity changes appeared to be constant following repeated exposure to hydrogen/air. This indicated that palladium films recover with no permanent damage or surface poisoning occurring from the hydrogen/air exposure at room temperature.

Fig. 2a, b and c shows the reflectivity change as a function of the hydrogen/air cycling for different concentrations corresponding to the three substrates. The black band on top of the figures corresponds to the time where the sample was exposed to hydrogen where as the white band corresponds to the air exposure. It is clear from the graphs in Fig. 2 that the palladium/silicon dioxide sample has the largest fractional reflectivity change $(\Delta R / R)$, followed by palladium/silicon nitride and finally the palladium/polycrystalline silicon sample. The fractional changes in reflectivity for all three substrate samples are summarized in Fig. 3. All samples display behav-
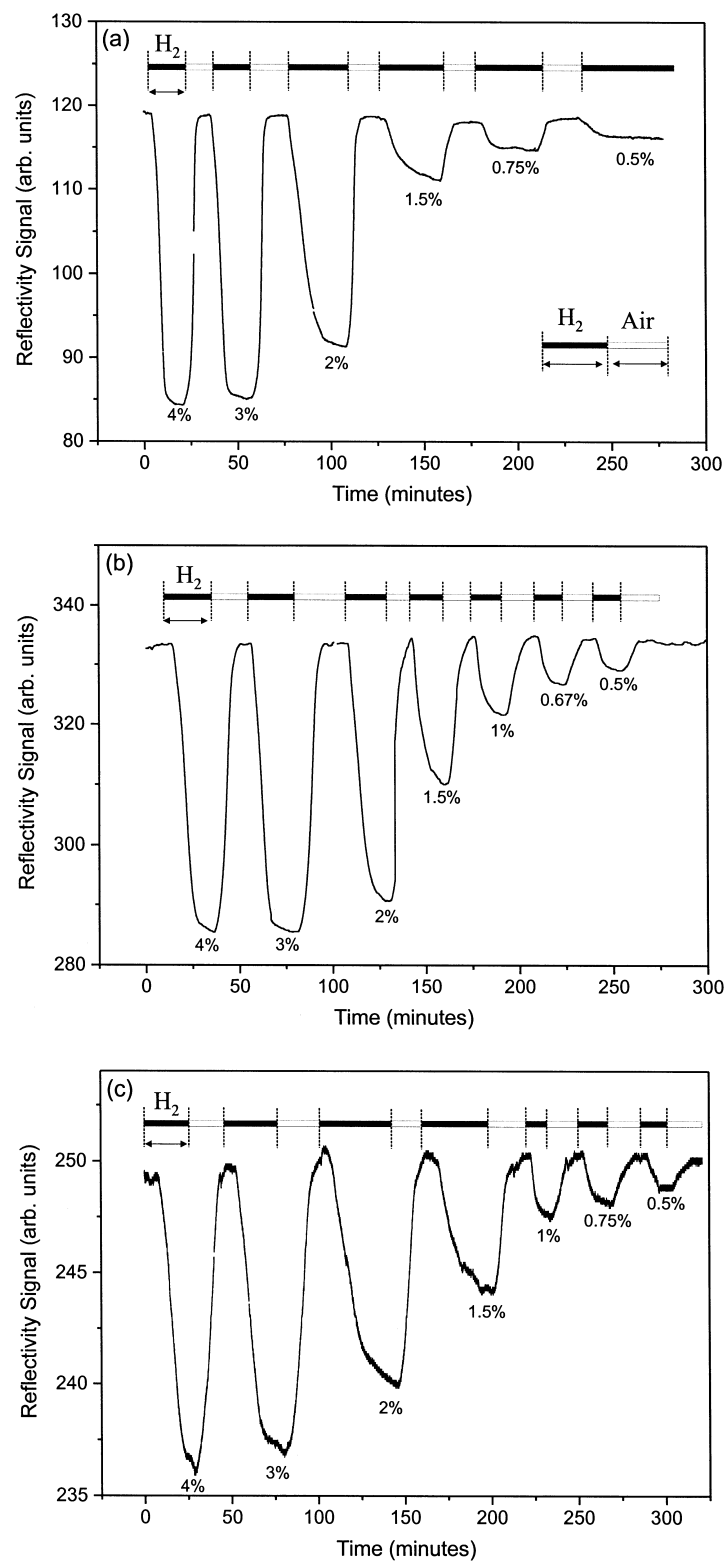

Fig. 2. Reflectivity signal of $8 \mathrm{~nm}$ palladium thin film exposed to hydrogen and air at different concentrations. The films were deposited on different substrates (a) silicon dioxide (b) silicon nitride and (c) polycrystalline silicon. 


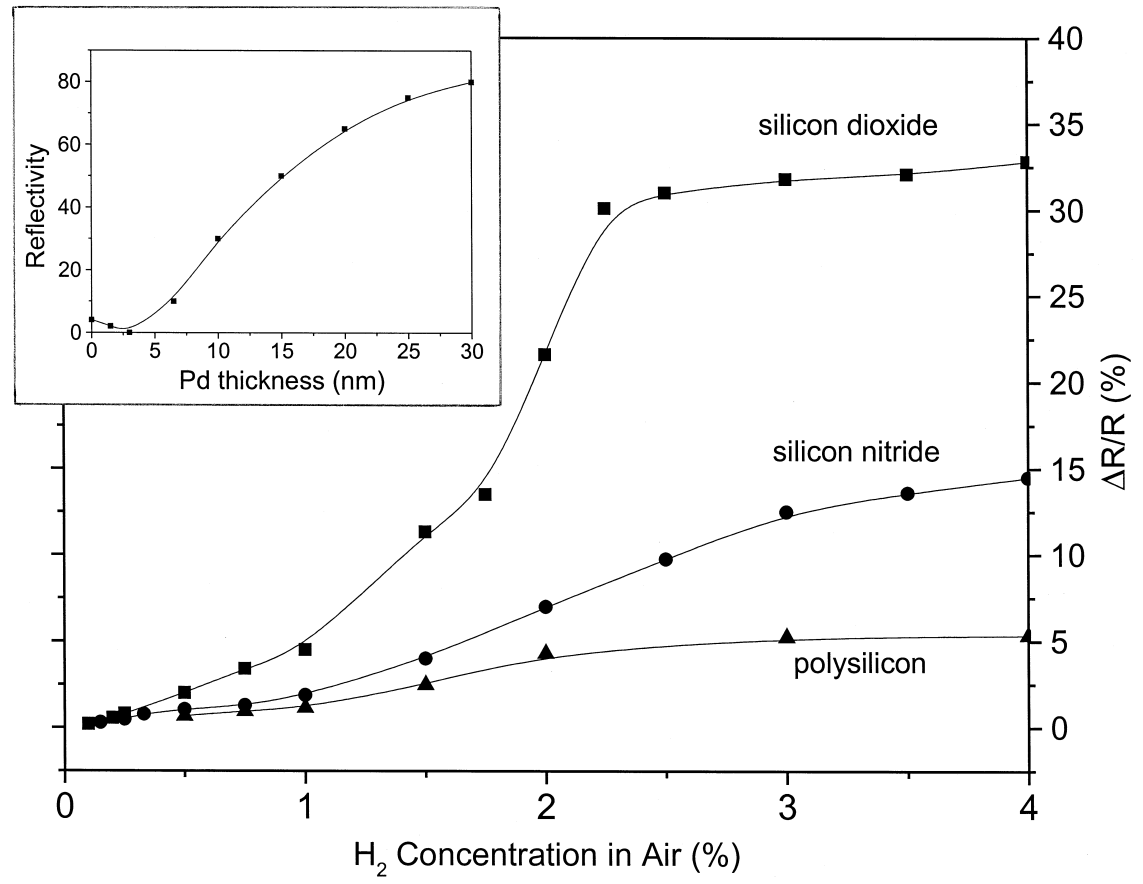

Fig. 3. Fractional reflectivity changes for different concentrations for the 8-nm palladium thin films on the different substrates. The upper left corner curve corresponds to fit data from absolute reflectivity measurements of different film thickness palladium samples on $\mathrm{SiO}_{2}$.

ior consistent with a mixed $\alpha / \beta$ phase as indicated by the change in response for a hydrogen concentration of $2 \%$. Typical absolute reflectivity data is also shown at the upper left corner as a function of film thickness (this fits to data from the different film thickness samples). This curve displays the characteristic minimum in reflectivity for optically thin metal films [5].

The fractional change in reflectivity reaches a plateau for all three substrate samples at exposure of highest hydrogen concentration $(3-4 \%)$. This plateau is approximately $33 \%$ for the silicon dioxide substrate, $15 \%$ for the silicon nitride and only $5 \%$ for the polycrystalline silicon. Similar curves have also been obtained for different palladium samples thickness. Specifically, samples ranging from 4 to $8 \mathrm{~nm}$ thickness appear to have the same fractional change, however 3-nm samples show a substantial increase in $\Delta R / R$ (up to $80 \%$ for $4 \%$ hydrogen exposure). This behaviour is the result of two effects occurring with Pd samples of decreasing thickness. The first corresponds to the decrease in the absolute reflectiv- ity (Fig. 3), and the second has to do with the penetration depth of the palladium samples at the probing laser wavelength (which is estimated to be approximately the same, using the bulk refractive index values of $\mathrm{Pd}[6])$. The fractional change in reflectivity depends on the amount of index of refraction change, which itself depends on the amount of hydrogen absorbed by the palladium. The observed signal however, depends on the amount of hydrogen seen by the probe beam. This in effect is the penetration volume of the laser beam in the palladium sample (assuming there is enough hydrogen to cover this volume). Clearly, the maximum reflectivity change will occur at the maximum penetration volume. A comparison of $8 \mathrm{~nm}(\Delta R / R=$ $33 \%)$ and $30 \mathrm{~nm}(\Delta R / R=9.2 \%) \mathrm{Pd}$ films on $\mathrm{SiO}_{2}$ (at $4 \% \mathrm{H}_{2}$ exposure) revealed a ratio of signals of $\sim 4$. Since the thickness of the samples is larger than the penetration depth, we make the assumption that $\Delta R$ is the same for both samples, then the observed difference is the ratio of the absolute reflectivities $(\sim 80 / 20=4)$. This supports the above reasoning. 
Film thickness changes due to lattice constant change from absorption of hydrogen (change from $\alpha$ to $\beta$ phase) may also contribute to changes in reflectivity. For the maximum hydrogen concentrations in this work $(4 \%)$, the ( $\mathrm{Pd}$ is considered to be in the mixed $\alpha / \beta$ phase) the lattice constant changes from 3.89 to $3.895 \AA$ [1]. This corresponds to $0.13 \%$ change in thickness. From the curve of absolute reflectivity as a function of thickness at the upper left corner of Fig. 3, it appears that the fractional change in reflectivity is negligible (estimated to be $<0.5 \%$ ) for the 8 -nm sample.

The observed difference in the fractional reflectivity change between the different substrates is believed to result from morphological changes of the palladium following exposure to hydrogen. This became clear when these samples were examined using
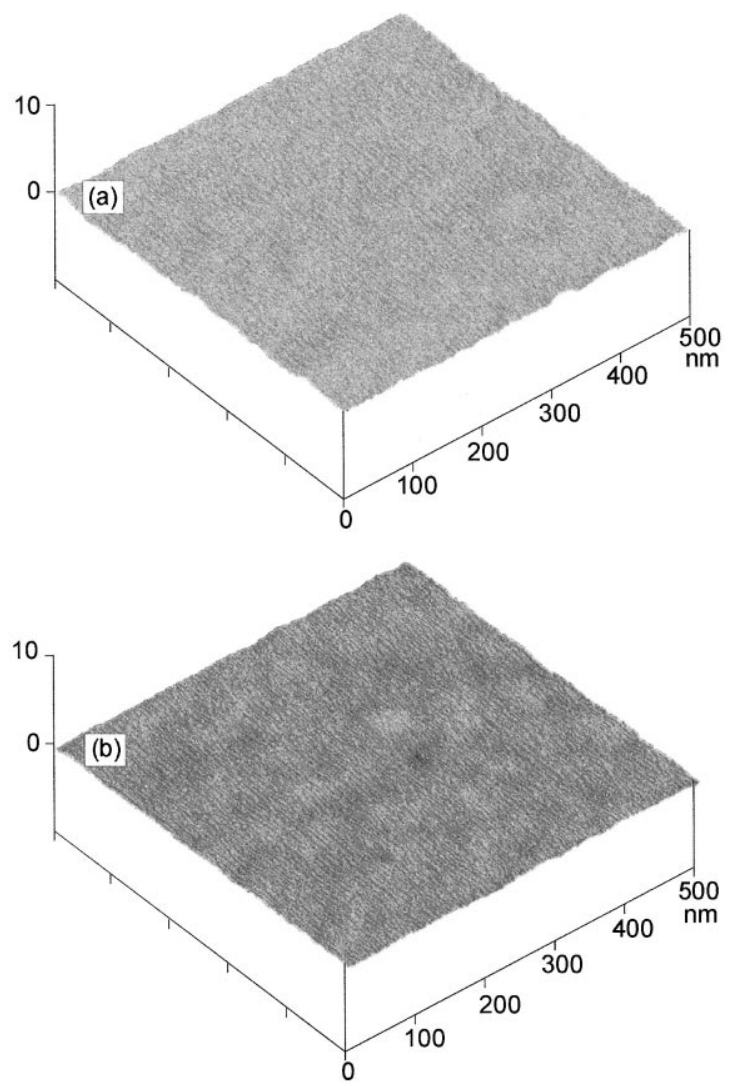

Fig. 4. AFM pictures of the $8 \mathrm{~nm}$ palladium film evaporated on silicon dioxide. (a) before (b) after hydrogen exposure.
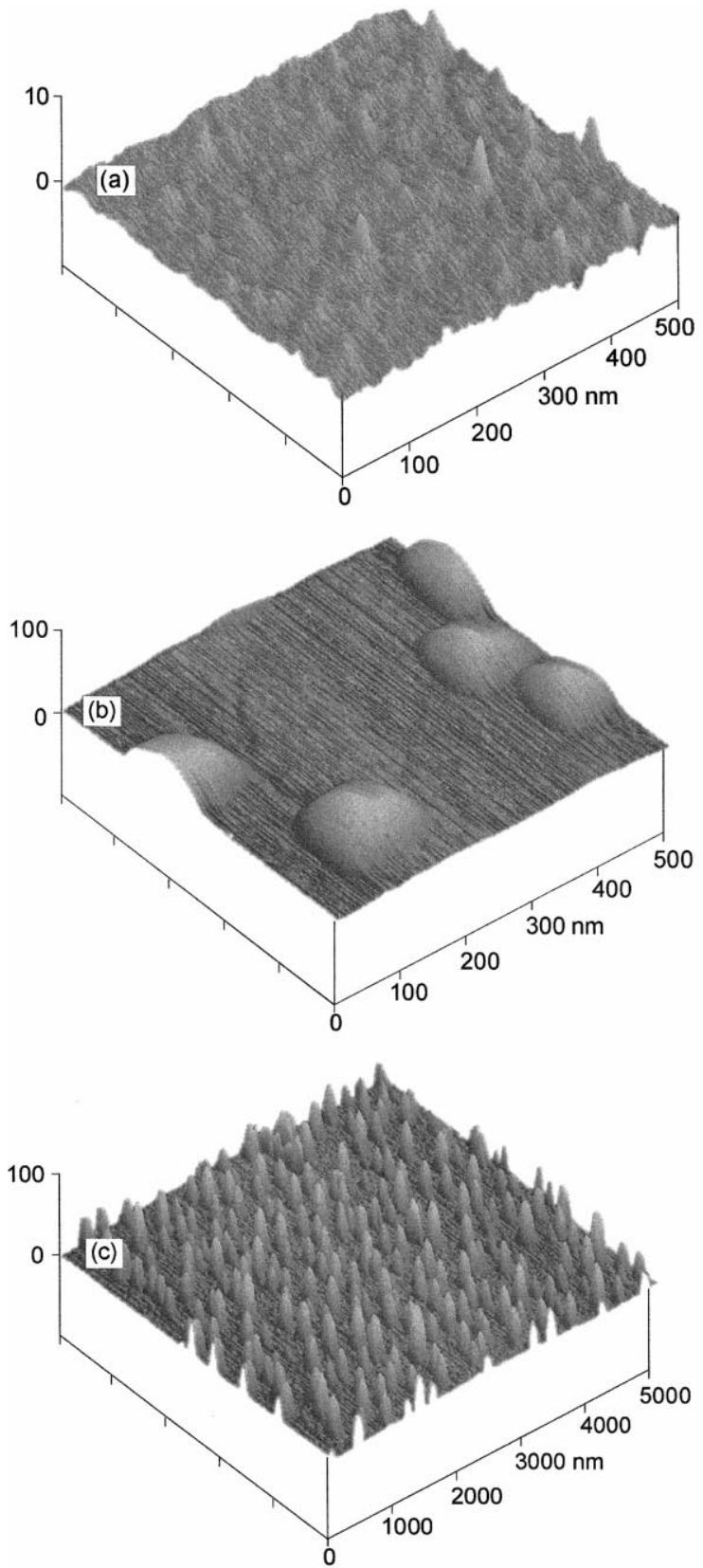

Fig. 5. AFM pictures of the $8 \mathrm{~nm}$ palladium film evaporated on silicon nitride. (a) before (b) after hydrogen exposure. (c) The same as (b) scanned over larger area $(5000 \mathrm{~nm})$.

near field microscopy [7]. Fig. 4 shows two AFM images of $8 \mathrm{~nm} \mathrm{Pd}$ evaporated on $\mathrm{SiO}_{2}$ covering an area of $500 \times 500 \mathrm{~nm}$. The first image (Fig. 4a) 
corresponds to the case where the Pd film was not exposed to hydrogen. No structure is apparent at this resolution, indicating a smooth and flat surface of $\mathrm{Pd}$ (a similar AFM image was observed for the substrate). The second image (Fig. 4b), corresponds to the case where the sample in Fig. 4a was exposed to hydrogen gas, and has developed small structural features with height of $<1 \mathrm{~nm}$.

Images from the 8-nm Pd samples with silicon nitride show more pronounced structural changes following hydrogen exposure. Fig. 5 shows three-dimensional AFM images of the 8-nm Pd film evaporated on the silicon nitride substrate. The first image (Fig. 5a) corresponds to the unexposed film. This surface is relatively flat with small $(<5 \mathrm{~nm})$ structural features. Image in Fig. 5b shows the Pd sample after it has been exposed to hydrogen. Clearly, the metal film forms structures with diameter of $\sim 100$ $\mathrm{nm}$ and height ranging between 80 and $100 \mathrm{~nm}$. This is more obvious in the images in Fig. $5 \mathrm{c}$ where a larger image area is shown $(\sim 5000 \mathrm{~nm})$. It should be pointed out that these images were collected after the hydrogen has escaped from the samples. Therefore, these morphological changes are considered permanent. The large nanometer structures formed from the Pd metal have on the average a thickness much larger than the penetration depth of the probe beam. This suggests that only a small fraction of the hydrogen absorbed by the Pd nanostructures will be detectable. This agrees with the reduced fractional reflectivity signal for this substrate in comparison with the silicon dioxide.

Finally, Fig. 6 also shows several AFM images for the 8-nm Pd film evaporated on polycrystalline silicon. Images in Fig. $6 a$ and $b$ are two-dimensional pictures of unexposed and exposed samples to hydrogen gas. The two dimension images use gray scale (seen to the side of the images) to indicate the height of the structures. The unexposed sample image indicates roughness of the order of $\sim 200 \mathrm{~nm}$. This roughness is characteristic of the polysilicon samples observed from AFM images of the substrate. The image of Fig. $6 \mathrm{~b}$ displays the Pd-polysilicon sample following exposure to hydrogen. It is not obvious from the picture that there has been any change. However, given the small signal in the reflectivity, we believe that Pd must be on in the form of structures (most probably on the already existing

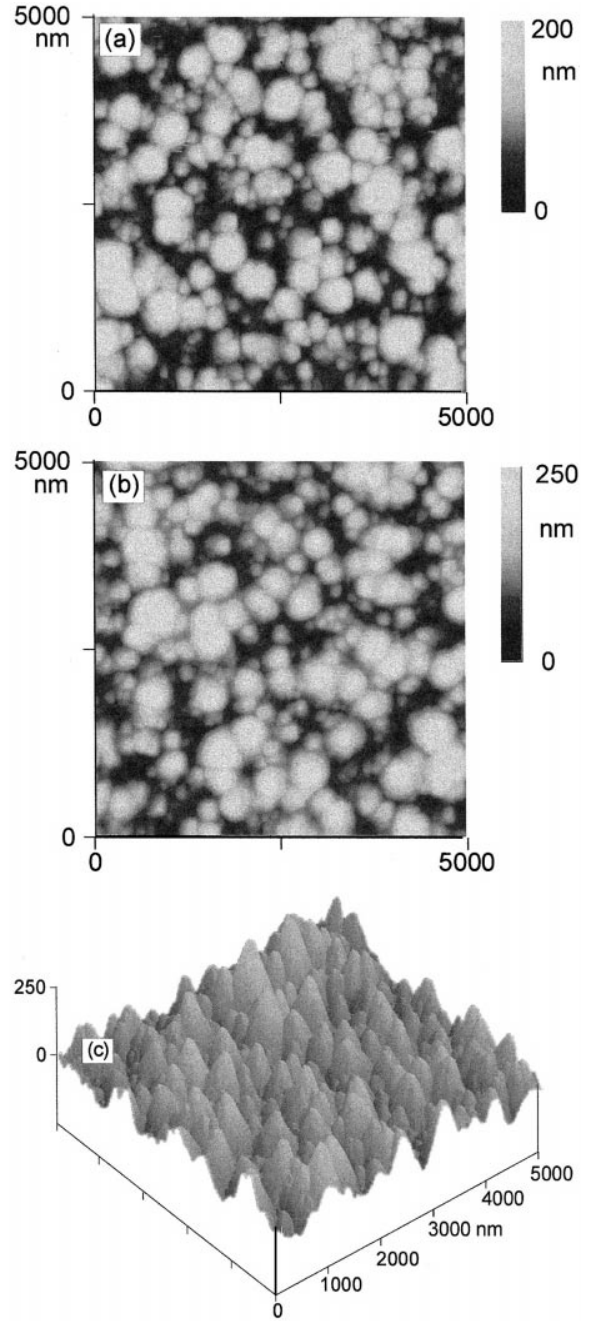

Fig. 6. AFM pictures of the 8-nm palladium film evaporated on polysilicon: (a) before (b) after hydrogen exposure. (c) The same as (b) but in three-dimensional form.

structures of the polycrystalline silicon) as is indicated by the small increase in the scale from $200 \mathrm{~nm}$ prior to exposure to $250 \mathrm{~nm}$ following exposure. Fig. $6 \mathrm{c}$ is a three-dimensional AFM image of the sample derived from Fig. $6 \mathrm{~b}$ shown for comparative purposes.

\section{Conclusion}

In conclusion, it appears that the best results in detecting hydrogen using Pd-optically thin films via 
changes in the index of refraction is by using $\mathrm{SiO}_{2}$ as the supporting structure. The critical factor in the modulated reflectance technique is the penetration depth at the probing wavelength of the metal. Thus, penetration-probing depth must be optimized with regard to the Pd film thickness.

Structural changes of the palladium film on the silicon nitride induced via exposure to hydrogen due to surface energy effects result to the creation of nanostructures. Although not very useful in hydrogen detection scheme, they may be considered useful in other areas of optoelectronics. Work is under way to study and possibly control the formation of these nanostructures and enhance some of their useful characteristics.

\section{References}

[1] F.A. Lewis, In the Palladium/Hydrogen System, Academic Press, New York, 1967.

[2] R. Jansson, H. Arwin, M. Armgarth, I. Lundstrom, Appl. Surf. Sci. 37 (1989) 44.

[3] K. Kalli, A. Othonos, C. Christofides, A. Lloyd Spetz, I. Lundstrom, Rev. Sci. Instrum. 69 (1998) 3331.

[4] A. Spetz, U. Helmersson, F. Enquist, M. Armgarth, I. Lundstrom, Thin Solid Films 177 (1989) 77.

[5] M.A. Butler, A.J. Ricco, Sensors and Actuators 19 (1989) 249.

[6] P.B. Johnson, R.W. Christy, Physical Review B 9 (1974) 5056.

[7] R. Erlandsson, M. Eriksson, L. Olsson, U. Helmersson, I. Lundström, L.-G. Petterson, J. Vac. Sci. Technol., B 9 (1991) 825. 